\title{
SÍNTESIS DE LA PREHISTORIA RECIENTE MADRILEÑA A PARTIR DE LA REVISIÓN DE LA COLECCIÓN BENTO ${ }^{1}$
}

\section{A SYNTHESIS OF THE PREHISTORY OF MADRID TROUGH THE REVISION OF THE BENTO COLLECTION}

\author{
por
}

\author{
Ma CONCEPCIÓN BLASCO BOSQUED \\ ELENA CARRIÓN SANTAFÉ
}

RESUMEN El trabajo ofrece una revisión de la Prehistoria Reciente de la Cuenca Baja del Manzanares que sintetiza el proceso histórico desde el Neolítico hasta el Bronce Final en el interior peninsular. Para ello se han reinterpretado los datos que ofrecen los numerosos yacimientos de la zona y algunos de los materiales más significativos depositados en el Museo Arqueológico de Barcelona, que forman parte de la Colección Bento.

\begin{abstract}
This paper offers a revision of the Recient Prehistory in Lover Manzanares Valley, with a syntesis of historical process from Neolithic to Final Bronze Age development in the Center of Iberian Peninsula. With this purpouse we have reinterpreted the data offered by the sites of the area, and most significative materials included in Bento Collection, stored in Archaeological Museum of Catalonia in Barcelona.
\end{abstract}

Palabras claves Neolítico, Edad del Bronce, Valle Manzanares

Key words $\quad$ Neolithic, Bronze Age, Manzanares Valley

La colección Bento es uno de los conjuntos de materiales prehistóricos madrileños más completos y citados en la Bibliografía científica, sin embargo, a partir del trabajo de Pérez de Barradas (1933-35) en el que se dan a conocer los yacimientos y algunos de los materiales recuperados en cada uno de ellos, sólo se hacen menciones a piezas puntuales, sin indicación de yacimiento al que pertenecen; además, la amplitud

1. Este trabajo se inscribe en el marco del Proyecto de Investigación financiado por la Dirección General de Investigación de la Comunidad de Madrid, referencia n ${ }^{\circ}$ 06/0180/2000 
cronológica de sus materiales permite obtener una síntesis renovada de la Prehistoria madrileña, desde el Paleolítico hasta el inicio de la Edad del Hierro, por ello merece la pena su estudio sistemático.

Tanto la forma en que se recuperaron los materiales, como la naturaleza de los mismos, así como el área de donde proceden y las características de los yacimientos, son paradigmáticas de cómo y dónde se han sentado las bases de la Prehistoria madrileña. Así pues, tanto la historiografía de la formación de esta colección como su propio contenido, pueden explicar las carencias y también la gran riqueza de la Prehistoria del Bajo Manzanares (Lámina 1), que por ser el tramo de cuenca más sobreexploatado de toda la Península, podría dar una idea del potencial arqueológico que pueden encerrar otras vegas fluviales, cuya reiteración y densidad de poblamiento pudo ser tan intensa, o más, que la del Manzanares. Debido a que todavía estamos en una etapa muy inicial de la revisión del inmenso volumen de materiales paleolíticos, hemos optado, para este homenaje al Prof. Vallespí, por ceñirnos a ofrecer una síntesis de la Prehistoria Reciente, primicia de lo que será el estudio completo y pormenorizado del conjunto. El trabajo ha sido posible gracias a las facilidades dadas por el Museo Arqueológico de Barcelona donde se custodia la mayor parte de la colección, a cuyo personal queremos agradecer su disponibilidad y ayuda.

\section{EL NEOLÍTICO Y EL COMIENZO DE LA COLONIZACIÓN DE LA CUENCA DEL MANZANARES}

Por lo que hoy sabemos, durante el Neolítico se mantiene la tradición de la ocupación de cuevas y abrigos localizados en las cabeceras de los ríos, en las estribaciones montañosas, concretamente en Madrid este modelo de asentamiento está representado por la Cueva del Aire (Fernández Posse 1980) y el Covacho de la Higuera (Rubio 1999-2000: 111) ambos en Patones en la Cuenca Alta del Jarama. Pero desde hace unos años, se ha confirmado que, paralelamente, se produce una colonización de las Cuencas Bajas, de la que es un buen ejemplo el Manzanares y el Jarama (Rubio 1999-2000) donde son numerosos los yacimientos que, en una pequeña superficie de las ocupaciones de la Edad del Bronce, ofrecen concentraciones de cerámicas neolíticas, frecuentemente con decoraciones incisas y superficies a la almagra.

Estos hallazgos se deben a la presencia de pequeñas instalaciones, probablemente de unas pocas unidades domésticas, incluso, de una única cabaña, que no dejan otro rastro que escasos elementos muebles amortizados. Desconocemos la extensión real de las ocupaciones y la duración de las mismas, pero la ausencia de formación de suelos y la carencia de estructuras e, incluso de grandes subestructuras de almacenaje, podrían hacer pensar en estacionamientos cortos, sin que podamos hacer más precisiones. Tampoco tenemos, hasta ahora, dataciones que nos permitan concretar el momento en que pudo iniciarse la atracción por las vegas bajas de los ríos, pero el fenómeno es paralelo al documentado en otras regiones peninsulares, como es el caso del País valenciano (Bernabeu y otros 1989).

En la colección Bento estos primeros agricultores y pastores de la Cuenca del Manzanares están representados en el yacimiento de Los Vascos, un poblado relativamente extenso, perteneciente a la etapa Protocogotas, localizado sobre un pequeño sitio Neolítico de cerámicas a la almagra de excelente calidad (Lámina 2, figuras 1 a 3). Se asienta en la margen derecha del Manzanares, al borde de la terraza inferior, en una ubicación que inauguran los grupos neolíticos y que se mantiene vigente hasta el Final de la Edad del Bronce en los inicios del Primer Milenio a. C. Está a unos 500 metros de la carretera nacional IV (Andalucía), a la altura del kilómetro 6, izquierda, en una zona que hoy se encuentra plenamente urbanizada. Además no se descarta que, en otro de los areneros prospectados por Bento, como el de Valdivia oeste, se produjera otro asentamiento Neolítico (Rubio 1999-2000: 110), al que podrían pertenecer los materiales reproducidos por Pérez de Barradas en su lámina XXXIII, 2 y 3 (Pérez de Barradas 1933-35). También 
en el arenero de Valdivia sobre la ocupación neolítica se instaló un hábitat del Bronce Final, perteneciente al Horizonte Cogotas I.

Estos sitios neolíticos no son excepcionales, sino que hay que ponerlos en relación con otros hallazgos similares situados aguas abajo del Manzanares, junto a los puntos kilométricos 3,500 y 3, 800 de la carretera de San Martín de la Vega donde las cerámicas neolíticas se dispersan por una escasa superficie de 1.600 y $260 \mathrm{~m}^{2}$ (Mercader y otros 1989:22) respectivamente, lo que confirmaría el reducido tamaño de los grupos. Otras intervenciones recientes confirman nuevos hallazgos en el curso inferior del río apuntando a que, ya en este momento, toda la vega baja era transitada por estas primeras poblaciones de agricultores y pastores que encontraron en ella un terreno muy favorable para la práctica de sus actividades productivas.

En el trabajo de Pérez de Barradas, no se hace referencia a la adscripción cronológica de los materiales neolíticos del arenero de Los Vascos, mientras que los fragmentos del arenero de Valdivia son clasificados como "cerámica indígena de la Primera Edad del Hierro" (Pérez de Barradas 1933-35: Lám. XXXIII).

\section{EL HORIZONTE CAMPANIFORME MADRILEÑO, UN REFERENTE EN LA PREHISTORIA PENINSULAR}

Aunque no se descarta la existencia de algún material calcolítico precampanifome entre los fragmentos cerámicos de la colección que nos ocupa, lo que no hay duda es de la presencia de dos fragmentos campaniformes que documentan sendos asentamientos entre los yacimientos prospectados y recolectados por Bento. Un dato que no resulta extraño si se tiene en cuenta la alta densidad de sitios pertenecientes a este horizonte en la Cuenca del Manzanares, aguas abajo de Madrid. Esta proliferación de hallazgos campaniformes se explica porque ésta es una de las pocas regiones peninsulares donde, además de los enterramientos, se han documentado lugares de hábitat de este Horizonte. Los dos yacimientos que han entregado sendos fragmentos de recipientes campaniformes son el Arenero de Santiago y, nuevamente el de Los Vascos.

La coincidencia de la ocupación Neolítica y campaniforme, con una posterior Protocogotas no es vanal, ya que se produce junto al lugar en el que Manzanares recibe uno de los cursos tributarios: el Arroyo Pradolongo y es precisamente en estos lugares en los que la abundancia de agua proporciona una mayor extensión de los humedales donde la ocupación es más intensa, quizás favorecida también por la facilidad de comunicación que ofrece el propio trazado fluvial.

Los hallazgos campaniformes corresponden a sendos cuencos de decoración incisa, tipo Ciempozuelos, pero desconocemos si estos materiales pertenecen a enterramientos o a lugares de hábitat, ya que nada nos dice Pérez de Barradas de su contexto y en ambos casos suelen aparecer en pequeñas concentraciones, correspondientes a una fosa aislada de inhumación individual o doble, o a un suelo de ocupación de escasas dimensiones, posiblemente como en el Neolítico, de una sola cabaña (Blasco ed. 1994). Incluso no descartamos que ambos fragmentos pertenezcan a un mismo yacimiento ya que Pérez de Barradas nos dice que estos dos areneros están muy próximos (Pérez de Barradas 1933-35: 26). Además, a poco más de un kilómetro de estos hallazgos se encuentra el yacimiento de Las Carolinas, lugar donde se recuperó un enterramiento con varias piezas campaniformes y, entre ellas, un cuenco con una singular decoración de soles y ciervos esquemáticos (Oberamier 1917 ; Blasco y Baena 1996), pertenecientes también al campaniforme inciso tipo Ciempozuelos. Tal concentración de hallazgos es llamativa y seguramente está provocada por la amplia vega que crea el Manzanares favorecido por el aporte de los arroyos Abroñigal y Pradolongo.

La importancia del Horizonte campaniforme en el bajo del Manzanares se completa con el yacimiento del Ventorro un asentamiento, situado a unos 5 kilómetros aguas abajo, donde se ha documentado la práctica de una primitiva metalurgia del cobre en un ambiente doméstico que emplea crisoles con decoración campaniforme (Priego y Quero 1992), actividad que también está testificada en Perales del Río, por la existencia 
de un recipiente-horno (Rovira 1989) encontrado entre los restos de un suelo de ocupación perteneciente a una única cabaña (Blasco y otros 1989), situada en la terraza baja, en posición similar al resto de los hallazgos campaniformes, a unos 3 kilómetros al sur del Ventorro.

El hallazgo previo del cementerio de Ciempozuelos permite a Pérez de Barradas, lo mismo que al resto de investigadores de la Prehistoria madrileña de las primeras décadas del siglo XX, la perfecta identificación de las cerámicas pertenecientes a este Horizonte.

\section{EL BRONCE PLENO EN LA COLECCIÓN BENTO: LA ESPADA DE LA PERLA SÍMBOLO DE PRESTIGIO}

Uno de los Horizontes que peor definidos estaban en la Cuenca del Manzanares era, precisamente el Bronce Pleno, posiblemente porque las cerámicas, obtenidas casi siempre en recogidas ocasionales, resultaban de difícil identificación ante la ausencia de ornamentaciones singulares, como ocurre en otros horizontes previos o posteriores como son el campaniforme o Cogotas I. Sin embargo ya Pérez de Barradas observa la similitud de algunos recipientes lisos, con los argáricos, adscribiéndolos a este círculo. Más tarde Almagro, al excavar el yacimiento sito en la Fábrica de Euskalduna, advierte también la existencia de ciertos rasgos cerámicos relacionables con materiales argáricos, como la existencia de una copa de pie alto (Almagro 1960b).

Pero, hasta el momento, el único yacimiento del Bronce Antiguo/Pleno excavado en cierta extensión es el del Tejar del Sastre (Quero 1982), precisamente uno de los puntos en los que prospecta el Sr. Bento, obteniendo "una hoja larga de sílex" y del que Pérez de Barradas dice que "en 1927 apareció una sepultura con el esqueleto de un niño en una tinaja, es probable que este yacimiento sea argárico" (Pérez de Barradas 1933-35: 48). Se trata de un extenso poblados de silos, entre cuyo espacio doméstico debieron de abrirse otros hoyos, similares a los de almacenamiento, para usarse como fosas de enterramiento, se localiza en torno al kilómetro 6 derecha de la carretera de Andalucía (nacional IV).

Muy próximo a este punto, a la izquierda de la mencionada vía, se encontraba el Arenero de La Perla, donde también intervino el Sr. Bento recuperando, además de varios recipientes lisos (Lámina 3, figuras 3 y 4), un magnífico ejemplar de espada de cobre (lámina 3, figura 1), calificada como argárica. Se trata, en efecto, de una pieza que responde al modelo de armas de esta categoría del Bronce Pleno peninsular (Almagro 1972), pero sus proporciones y robustez la enmarcan dentro de las armas más septentrionales, recuperadas en el área cantábrica y la Meseta Norte, mostrando ciertos paralelos con la metalurgia atlántica. Tampoco en este caso conocemos el contexto del hallazgo, pero fuera una pieza de ajuar funerario, o un depósito aislado o no, la presencia de este ejemplar habla de la existencia de símbolos de prestigio vinculables a determinados personajes de la élite. Si todavía en estos momentos la posesión de un arma de esta categoría, en cuya confección se amortiza una gran cantidad de metal, representa una notoriedad importante, el propietario de la espada, de 730 gramos, acumulaba en este arma más metal que la mayoría de las tumbas argáricas con una panoplia completa y duplica e incluso triplicaba el peso de la mayoría de las espadas del sudeste.

No lejos de este yacimiento Tejar del Sastre/La Perla, posiblemente un único asentamiento de cierta extensión, recuperó el Sr. Bento otras piezas cerámicas lisas que podrían pertenecer a hábitats del mismo horizonte, concretamente en los areneros de Quitapenas (Lámina 3, figura 2, Orcasitas y Las Mercedes (Lámina 3, figuras 5 y 6), este último no citado en el trabajo de Pérez de Barradas. Estos posibles establecimientos unidos al existente en la Fábrica de Euskalduna, excavado posteriormente por Almagro, certifican una alta concentración de poblamiento durante el Bronce Antiguo, en torno a la confluencia del Pradolongo con el Manzanares, no sólo por el número de sitios, sino también por el mayor tamaño de los mismos, ya que alguno de ellos podría alcanzar una superficie superior a una Ha.. Aunque no hay documentación de las estructuras domésticas, ahora sí son perfectamente reconocibles abundantes hoyos, muchos de ellos 
silos, dispersos en toda la superficie habitada, que, en algunos casos, pudieron tener la función de fosas de enterramiento. Los poblados de estas características van a perpetuarse a lo largo de toda la Edad del Bronce, hasta el final del Horizonte Cogotas I, desapareciendo en el Hierro Antiguo.

La mayor extensión de los hábitats y el gran volumen de contenedores para almacenamiento de excedentes, podrían indicar ciertos cambios en el tamaño de los grupos y, posiblemente también en la duración de los estacionamientos, pero la reiterada ausencia de suelos de ocupación nos ha privado de una información más precisa sobre la estructura social de estos grupos y sobre las pautas de sus desplazamientos, aunque sí se observa en la mayoría de estos sitios, entre ellos, en el Tejar del Sastre, que existen abandonos y reocupaciones de los mismos lugares, lo que provoca la superposición de algunos de los hoyos (Quero 1982), y aunque desconocemos la distancia temporal entre cada uno de estos establecimientos, sabemos que algunos podrían estar relativamente próximos pues corresponden a grupos del mismo entorno cultural, mientras que otros se distancian varias centurias. En el caso del yacimiento que nos ocupa, existió una primera ocupación campaniforme y, tras el abandono de los grupos de Bronce Antiguo, se produjo la presencia, también puntual, de gentes del Horizonte Protocogotas.

Los datos con los que contamos nos permiten atisbar que el campaniforme y el Bronce Antiguo, de cerámicas lisas, cubren un lapso temporal desde fines del III milenio hasta mediados del II milenio, en paralelo a lo que ocurre en otras áreas peninsulares. Así mismo, pese a mostrar un cierto conservadurismo en las pautas y modelos de hábitats, tanto la tecnología como los cambios formales e ideológicos son asimilados con rapidez, evidenciando una permanente red de contactos con otros círculos peninsulares y extrapeninsulares.

\section{LA SECUENCIA PROTOCOGOTAS-COGOTAS I EN EL BAJO MANZANARES: TRADICIÓN Y RENOVACIÓN}

La segunda mitad del Segundo milenio a. C. y el comienzo del Primero, están caracterizados por la secuencia Protocogotas-Cogotas I, hoy perfectamente definida y bien representada en la colección Bento ya que contiene, entre sus materiales, uno de los lotes más completos y significativos de la etapa Protocogotas procedente en su mayor parte del yacimiento de Los Vascos (Lámina 4) y, posiblemente también, de Valdivia oeste, además de algunos de los ejemplares más singulares del Horizonte Cogotas I final.

En el arenero de los Vascos, tras la presencia puntual de grupos neolíticos y campaniformes, se produce una extensa ocupación Protocogotas cuyos hábitats, a la luz de la información deparada por el cercano yacimiento de Caserío de Perales, son muy similares a los del Bronce Antiguo, con numerosos "silos" y algunas fosas de enterramiento dispersas entre la superficie habitada. Entre las novedades hay que destacar el gusto por la cerámica ornamentada con incisiones e impresiones, que desarrolla monótonos y repetitivos temas de zig-zags, espigas o amplios triángulos y círculos, siempre en frisos seguidos y sobre cazuelas que mantienen acusadas carenas y fondos convexos, como en la etapa anterior, aunque la línea de carenación tiende a situarse más cerca de la boca (Pérez de Barradas 1933-35: Lámina XXXII) y, en algunos ejemplares, la decoración está trazada con una línea discontinua que marca el precedente de lo que será la técnica de boquique (Lámina 4, figura 3).

Aunque la colección que nos ocupa no tiene materiales metálicos de este horizonte, los datos procedentes del cercano yacimiento del Caserío de Perales, hablan de la práctica de una metalurgia de bronces binarios para fundir piezas de tipologías propias del Bronce Pleno, caso de los puñales de remaches o las puntas de flecha de pedúnculo prolongado. Es posible que existiera un comercio de objetos metálicos, pero en estos yacimientos, como en los campaniformes y en los del Bronce Antiguo, siguen fundiéndose pequeñas piezas y se procesa el mineral en recipientes horno, dentro del ámbito doméstico. 
Otro de los areneros prospectado por Bento que puede tener una ocupación de este horizonte es el del Cojo, según se desprende de la decoración de un recipiente troncocónico, ornamentado con diseños realizados con finas incisiones trazadas con el barro con textura cuero (Lámina 4, figura 5).

A partir de mediados del siglo XIII a. C., se observa una importante renovación que, aunque aparentemente no parece acusarse tanto en los repertorios cerámicos, sí va a desembocar en la introducción de novedades en todos los órdenes. Nos estamos refiriendo al Horizonte Cogotas I, que cubre el Bronce Final de la zona y que, incluso, en sus fases más recientes, bien representadas en la Colección Bento, llegó a ser coetáneo del Hallsttat Antiguo, a juzgar por algunos paralelos morfológicos y ornamentales.

Los yacimientos de este conjunto que mejor representan esta etapa son los Areneros de Valdivia y Martínez, con un importante repertorio vascular que ha sido tradicionalmente utilizado como ejemplo de las características cerámicas excisas de este círculo (Molina y Arteaga 1976), aunque sin especificar su procedencia exacta, pues sólo se indica: Areneros del Manzanares. El arenero Martínez proporcionó también materiales metálicos, pues como indica Pérez de Barradas: "De bronce ha recogido el dicho señor un punzón de sección rectangular, un trozo de vaso y un cuchillo curvo," (Pérez de Barradas 1933-35: 52).

Parece que también en estos dos yacimientos del Horizonte Cogotas I de plenitud, existieron asentamientos previos, concretamente en el de Martínez, se habla de la existencia de "fondos de cabaña" con material lítico asociado a cerámicas lisas, que podrían ser Calcolíticas o del Bronce Antiguo, mientras que en Valdivia pudo haber, además de una ocupación Neolítica, algún asentamiento Protocogotas, por lo que vuelve a repetirse la reiterada ocupación de un sitio a lo largo de un dilatada período de tiempo, con momentos de abandono que no descartamos pudieran ser prolongados.

Por la información disponible, los yacimientos de este Horizonte mantienen características similares a las de los grupos predecesores de la E. del Bronce, pero ahora el volumen de los "hoyos" o silos tiende a reducirse y, hasta ahora, ninguno de los asentamientos de este momento de la Región de Madrid, ha proporcionado enterramientos, aunque sí algunos restos óseos humanos, dispersos entre desechos domésticos y faltan o son más raros los enterramientos de animales completos, quizás ofrendas, que se documentan en momentos previos. Así pues se constata un notorio enrarecimiento de los enterramientos que indica un cambio ideológico importante.

Desde el punto de vista de la metalurgia, a falta de los datos que puedan proporcionarnos la analítica del cuchillo del arenero Martínez, se confirma el comienzo de las aleaciones ternarias en la zona, un dato que podría hablar, como en horizontes previos, de ciertas vinculaciones con la metalurgia atlántica. Este hecho está ligado a la presencia de tipos novedosos, como las primeras fíbulas, concretamente un ejemplar de codo, de morfología sícula, que apunta a un comercio, posiblemente indirecto, con el Mediterráneo. Otra novedad la proporciona la propia colección Bento, con un cuchillo de hoja curva en bronce (Lámina 5.2), prototipo de los que se fundirán en hierro. En la Península, frente a la abundancia de cuchillos afalcatados de hierro, son raros los fundidos en bronce, la excepción la constituye una hoja procedente de Agullana y un cuchillo completo con enmangue y hoja en bronce que procede de Tarragona (Ruiz Zapatero 1983: 899-900).

Pero las piezas que más se han representado de esta colección son algunos ejemplares cerámicos de estos yacimientos. Concretamente del Arenero Martínez procede un gran vaso restaurado (Lámina 5.1), de acusado galbo y pie plano y estrecho con decoración de guirnaldas realizadas en boquique y línea cosida en la boca; fue clasificada por Pérez de Barradas como "urna celta" y recogida por M. Almagro como "Procedente de los areneros del Valle del Manzanares" (Almagro 1960a: 222), a partir de esta publicación es tomado como ejemplo de la técnica de boquique citándose siempre con idéntica procedencia inespecífica (Fernández Posse 1982: 155, fig. 3.1 y 1986: 482, fig 3, 17). Se trata de un ejemplar muy característico de los momentos epigonales de Cogotas I y su morfología y diseño guardan paralelos con producciones del horizonte austro-bávaro del Hallsttat C/D (Werner 1987: vol. III: figs.50 y 52, pp. 34 y 36), y aunque el recipiente madrileño presenta la decoración realizada con técnica de boquique, singular del Horizonte 
Cogotas I, sus trazos discontinuos recuerdan a los de la ruedecilla utilizada también por algunos grupos del Hallsttat centroeuropeo (Werner 1987, vol. II: 26), sin olvidar el posible origen local de esta técnica, con precedentes claros en la etapa Protocogotas (Lámina 4.3).

De cronología relativamente similar a la del arenero Martínez son los ejemplares cerámicos del Arenero de Valdivia Centro (Lámina 5, 3 a 6) que también han sido reproducidos en diversos trabajos, sin más referencia que su procedencia del Valle del Manzanares (Molina y Arteaga 1976). Las piezas 3, 5 y 6 de la lámina 5 son algunos de los mejores exponentes del virtuosismo que la excisión alcanza en este Horizonte, técnica que, a veces, se aplica sobre formas bastante ajenas a la tradición local, como es el caso del soporte/carrete (Lámina 5.3), con paralelos en el yacimiento burgalés del Alto de Yecla, Silos (Almagro 1960a: 209, fig. 181), imitando quizás las piezas de este tipo que, a partir del denominado Bronce Final II de Molina, son frecuentes en el sur peninsular y, en especial, en el Bronce Final Tartésico (Molina 1978: 219-220), aunque siempre carentes de decoración.

Este horizonte tardío de Cogotas I representado en la Colección Bento por los Areneros Martínez y Valdivia, hunde sus raíces en la tradición local, pero con el transcurso del tiempo se abre a influjos múltiples, constatado por la tecnología y tipos metálicos, pero también por la morfología de sus recipientes más cuidados, muy cercanos a las modas continentales, en paralelo con los motivos decorativos que desarrollan de guirnaldas, dobles hachas, ajedrezados, e incluso por el empleo de pigmentos rojos que se embuten después de la cocción de las cerámicas proporcionando un cromatismo similar al de la pintura post cocción coetánea.

Con el final de Cogotas I desaparecen las grandes concentraciones de "hoyos" o silos que favorecen la acumulación de desechos por lo que se hace más difícil la localización de los débiles suelos del Hierro I, además, se produce un cierto alejamiento de los cauces de los ríos para buscar las terrazas más altas o, incluso los cerros y espolones de la orilla izquierda del río. Teniendo en cuenta el área de intervención del Sr. Bento, estas circunstancias explican la ausencia de materiales de la Edad del Hierro que, en la Prehistoria madrileña, marca una clara ruptura con la larga etapa de ocupación de las zonas más próximas a las vegas.

\section{BIBLIOGRAFÍA}

ALMAGRO BASCH, M. (1960a): "Los campos de urnas de España”, Cap. III de Historia de España. España Protohistórica. Vol I, 2: 141-240, dirigida por R. Menéndez Pidal. Madrid.

(1960b): "Hallazgos arqueológicos en Villaverde", Memorias de Museos y Archivos municipales XVI a XIII: 5-29. Madrid.

ALMAGRO GORBEA, M. (1972): "La espada de Guadalajara y sus paralelos peninsulares", Trabajos de Prehistoria 29: 55-82. Madrid.

BERNABEU, J.; GUITART, I. y PASCUAL J.L. (1989): "Reflexiones en torno al patrón de asentamiento en el País valenciano entre el Neolítico y la edad del Bronce", Saguntum 22: 99-122.Valencia.

BLASCO, M ${ }^{a}$ C., ed. (1994): El Horizonte campaniforme de la Región de Madrid en el centenario de Ciempozuelos. Universidad Autónoma de Madrid.

BLASCO, Mㄹ C. y BAENA J. (1996): "El yacimiento de Las Carolinas y la cerámica simbólica campaniforme. Algunos datos para su interpretación", en MOURE, A. (ed.): "El Hombre Fósil” 80 años después: 417-446. Univ. de Cantabria, Santander.

BLASCO, MaC.; CAPRILE, P.; CALLE, J. y SÁNCHEZ-CAPILLA, Ma L. (1989): "Yacimiento campaniforme en el Valle del Manzanares (Perales del Río, Getafe-Madrid)", Estudios de Prehistoria y Arqueología madrileñas 1989: 83-114. Ayuntamiento de Madrid. 
FERNÁNDEZ POSSE, Ma D. (1980): "Los materiales de la Cueva del Aire”, Noticiario Arqueológico Hispánico 10: 41-64. Madrid.

_ (1982): "Consideraciones sobre la técnica de boquique", Trabajos de Prehistoria 39: 137-159. Madrid. (1986): "La Cultura de Cogotas I", Homenaje a Luis Siret (1934-1984): 475-487. Junta de Andalucía Ministerio de Cultura.

MERCADER, J.; CORTÉS, A. F. y GARCÍA, Ma E. (1989): "Nuevos yacimientos neolíticos y de la Edad del Bronce en el término municipal de Madrid", Estudios de Prehistoria y Arqueología madrileñas 1989: 21-81. Ayuntamiento de Madrid.

MOLINA, F. (1978): "Definición y sistematización del Bronce Tardío y Final en el Sudeste de la Península Ibérica”, Cuadernos de Prehistoria de la Universidad de Granada 3: 159-232.

MOLINA, F. y ARTEAGA, O. (1976): "Problemática y diferenciación en grupos de la cerámica con decoración excisa en la Península Ibérica", Cuadernos de Prehistoria de la Universidad de Granada 1: 175-214.

OBERMAIER, H. (1917): "Yacimiento prehistórico de Las Carolinas", Memoria de la Comisión de Investigaciones prehistóricas y Paleontológicas 16. Madrid.

PÉREZDE BARRADAS, J.(1933-35): “Nuevos estudios sobre Prehistoria madrileña”, Anuario de Prehistoria madrileña 46: 3-90. Madrid.

PRIEGO, Ma C. y QUERO, S.(1992): "El Ventorro un poblado prehistórico en los albores de la metalurgia", Estudios de Prehistoria y Arqueología madrileñas 8. Ayuntamiento de Madrid.

QUERO, S. (1982): "El poblado del Bronce Medio del Tejar del Sastre (Madrid)", Estudios de Prehistoria y Arqueología madrileñas 1982: 184-247. Ayuntamiento de Madrid.

ROVIRA, S. (1989): "Recientes aportaciones para el conocimiento de la metalurgia primitiva en la provincia de Madrid: Un yacimiento campaniforme en Perales del Río (Getafe, Madrid)", Actas del XIX CNA, vol I: 355-367. Zaragoza.

RUBIO, I. (1999-2000): "Las Primeras Sociedades agrícolas en Madrid. Neolítico y Calcolítico Campaniforme", en La Arqueología madrileña en el final del siglo XX: desde la Prehistoria hasta el año 2000. Boletín de la Asociación española de amigos de la Arqueología 39-40.

RUIZZAPATERO, G. (1983): Los Campos de Urnas del NE. de la Península Ibérica. (2 tomos). Universidad Complutense. Madrid.

WERNER, S. (1987): El Bronce Final y la Primera Edad del Hierro en Centroeuropa", (3 vols.) Universidad Autónoma de Madrid. 


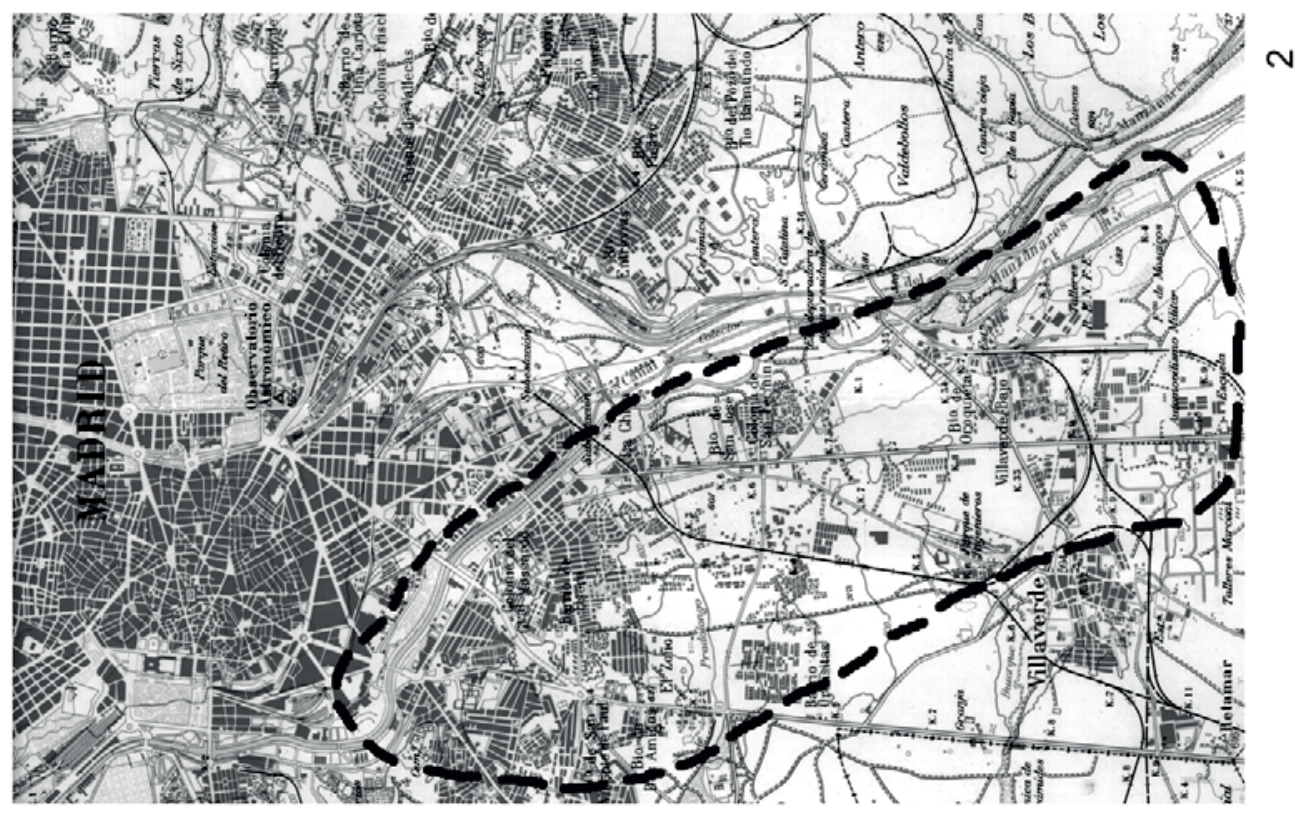

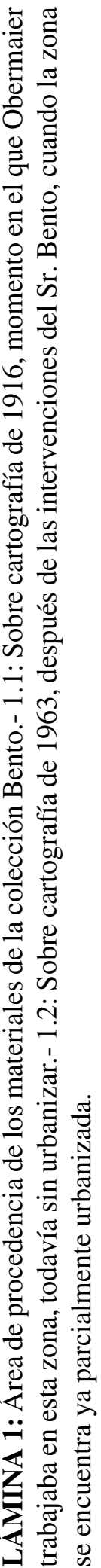

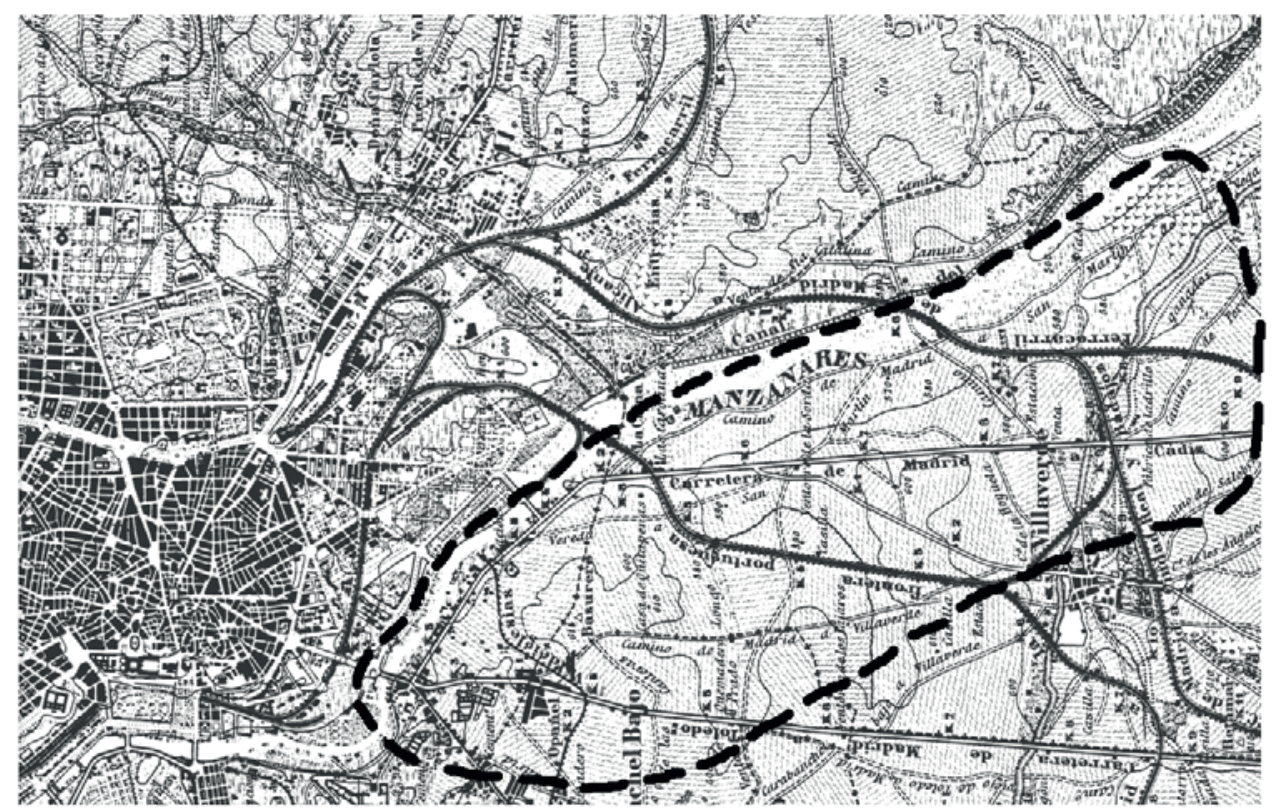



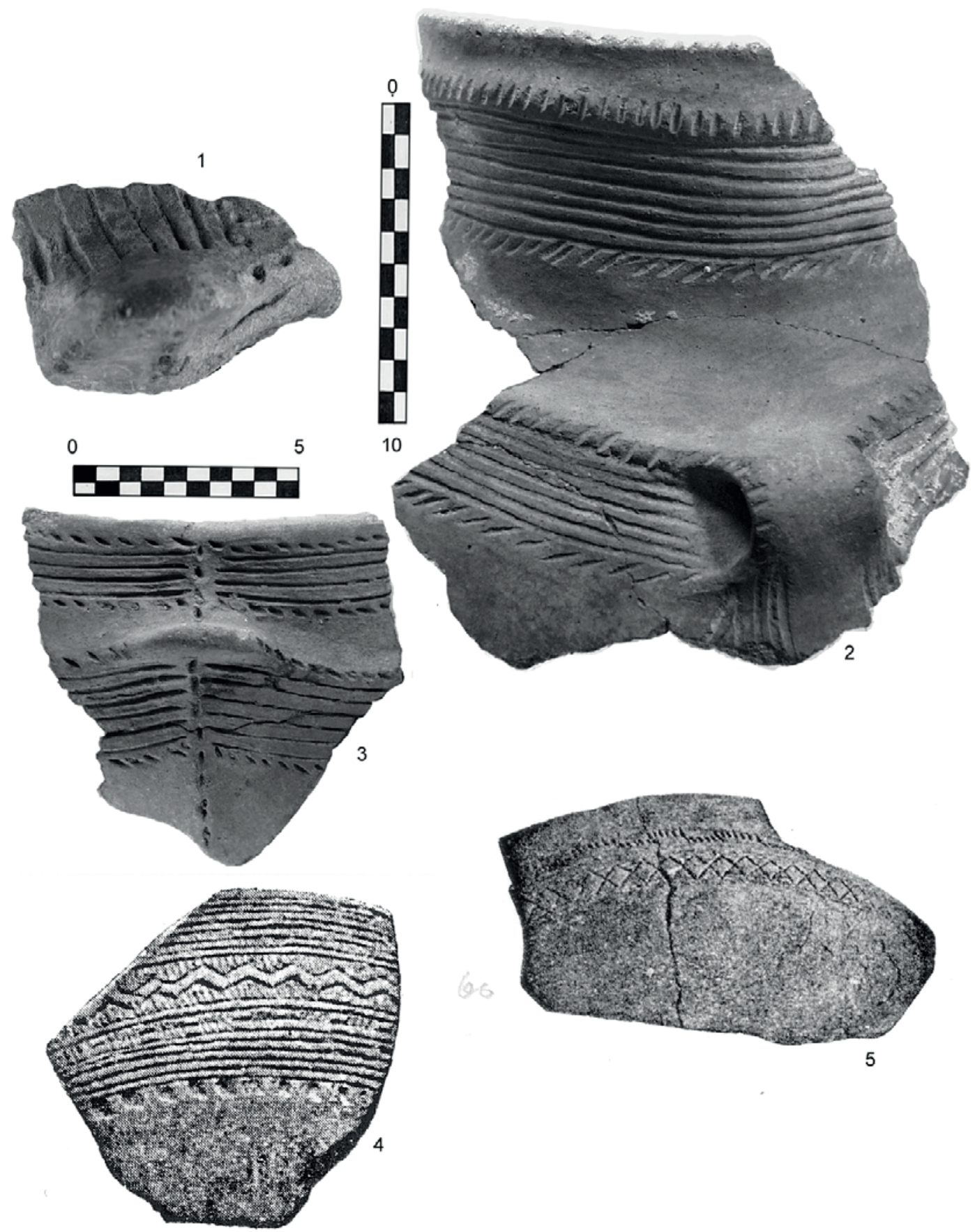

LÁMINA 2: Cerámicas neolíticas y campaniformes de la Colección Bento (Museo Arqueológico de Barcelona, MAC). 1 a 3: Materiales neolíticos procedentes del Arenero de los Vascos. 4: Fragmento de cuenco campaniforme inciso del Arenero de los Vascos (según Pérez de Barradas 1933-35: lám. XXXII.1). 5: Fragmento de cazuela campaniforme con decoración incisa, arenero de Santiago (según Pérez de Barradas 1933-35: lám. XXXIII.1). 


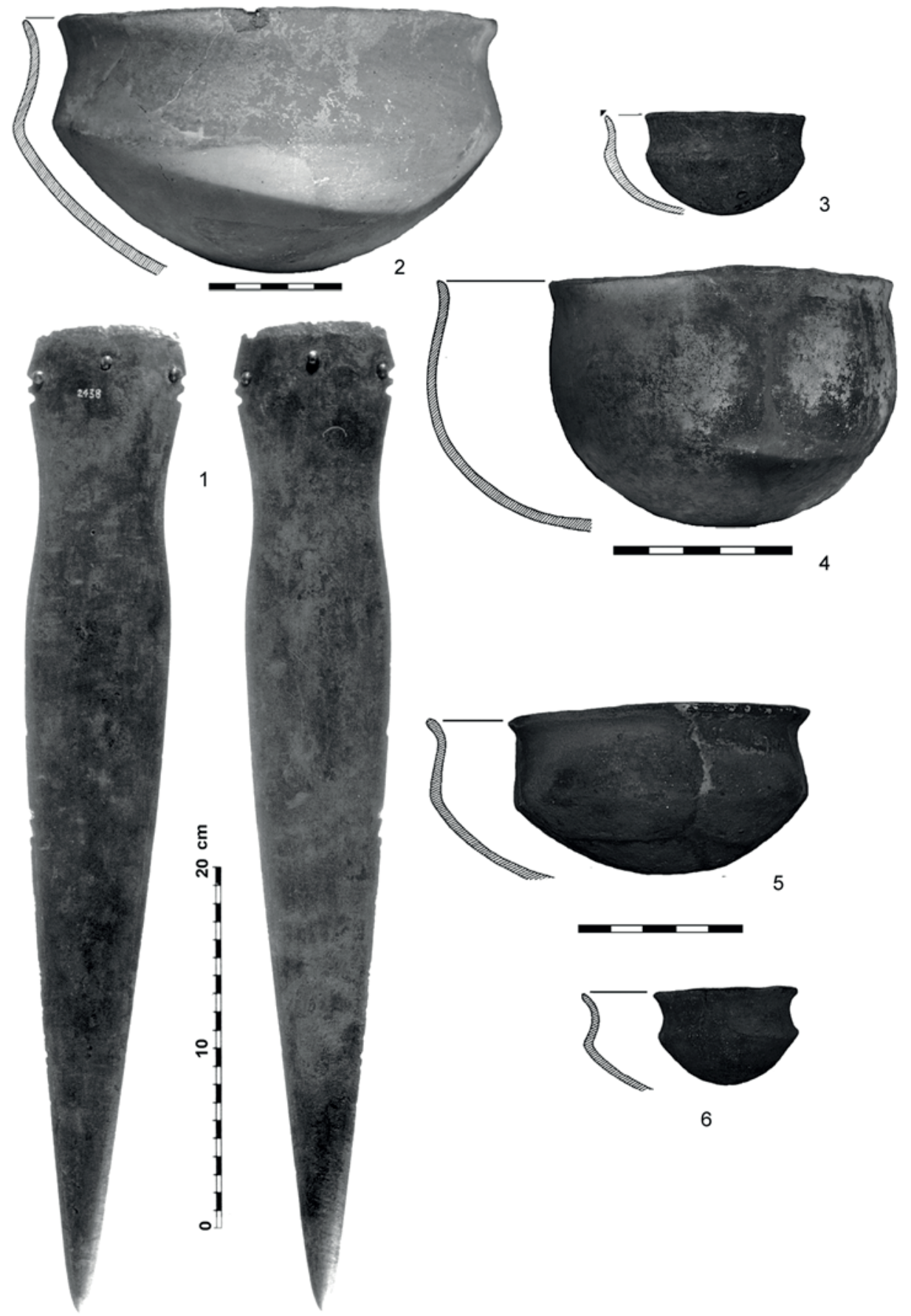

LÁMINA 3: Materiales del Bronce Antiguo pertenecientes a la colección Bento (Museo Arqueológico de Barcelona, MAC). 1: Recipiente cerámico, Arenero Quitapenas. 2: Espada de cobre del arenero de La Perla (Foto archivo MAC). 3 y 4 recipientes del arenero de La Perla. 5 y 6: Vasijas del Arenero de Las Mercedes.

ISSN: 1133-4525 ISSN-e: 2255-3924

SPAL 9 (2000)

http://dx.doi.org/10.12795/spal.2000.i9.17 

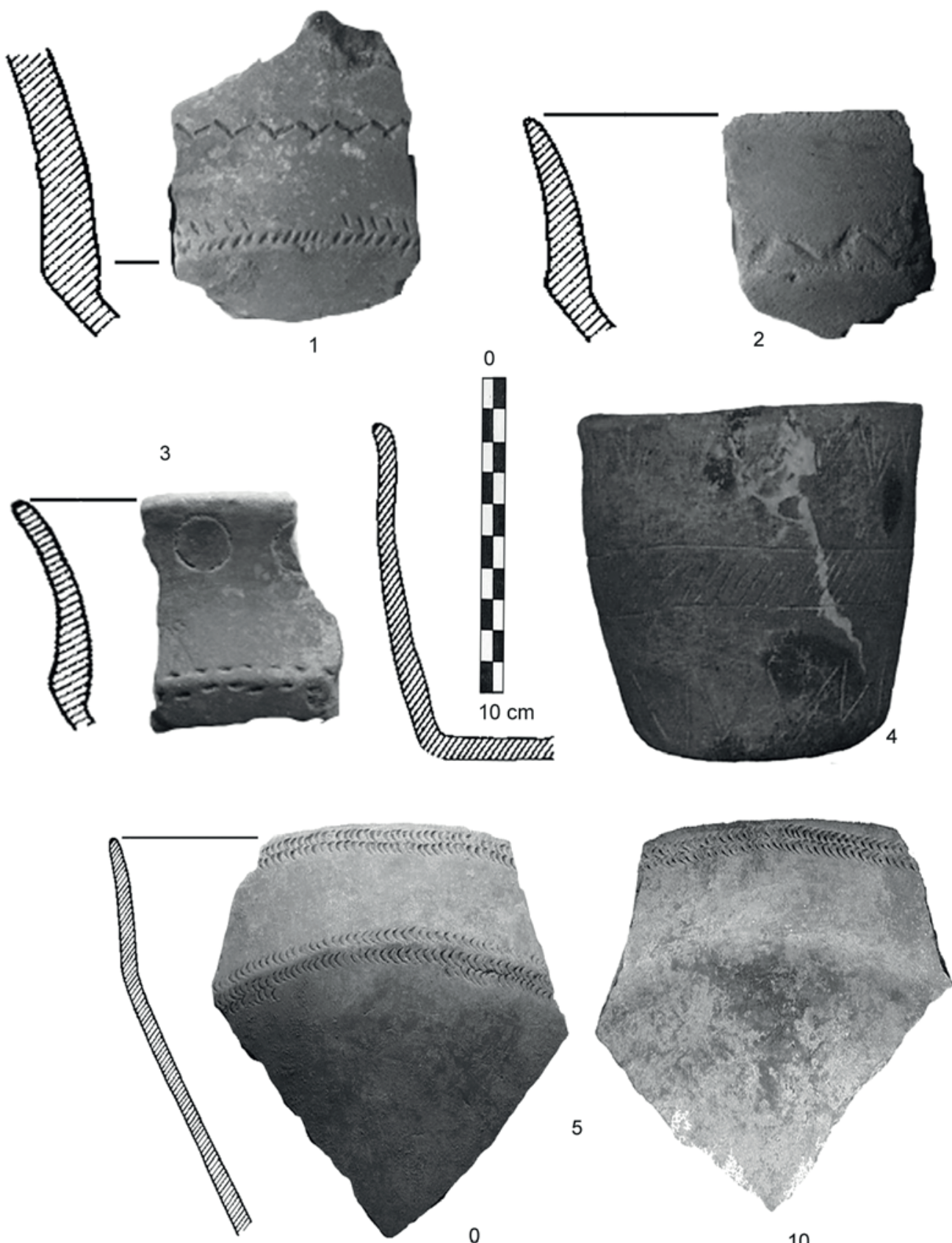

10

LÁMINA 4: Cerámicas decoradas representativas del Horizonte Protocogotas pertenecientes a la Colección Bento (Museo Arqueológico de Barcelona, MAC). 1 a 3 y 5: Fragmentos procedentes del Arenero de Los Vascos. 4: Recipiente obtenido en Arenero del Cojo. 

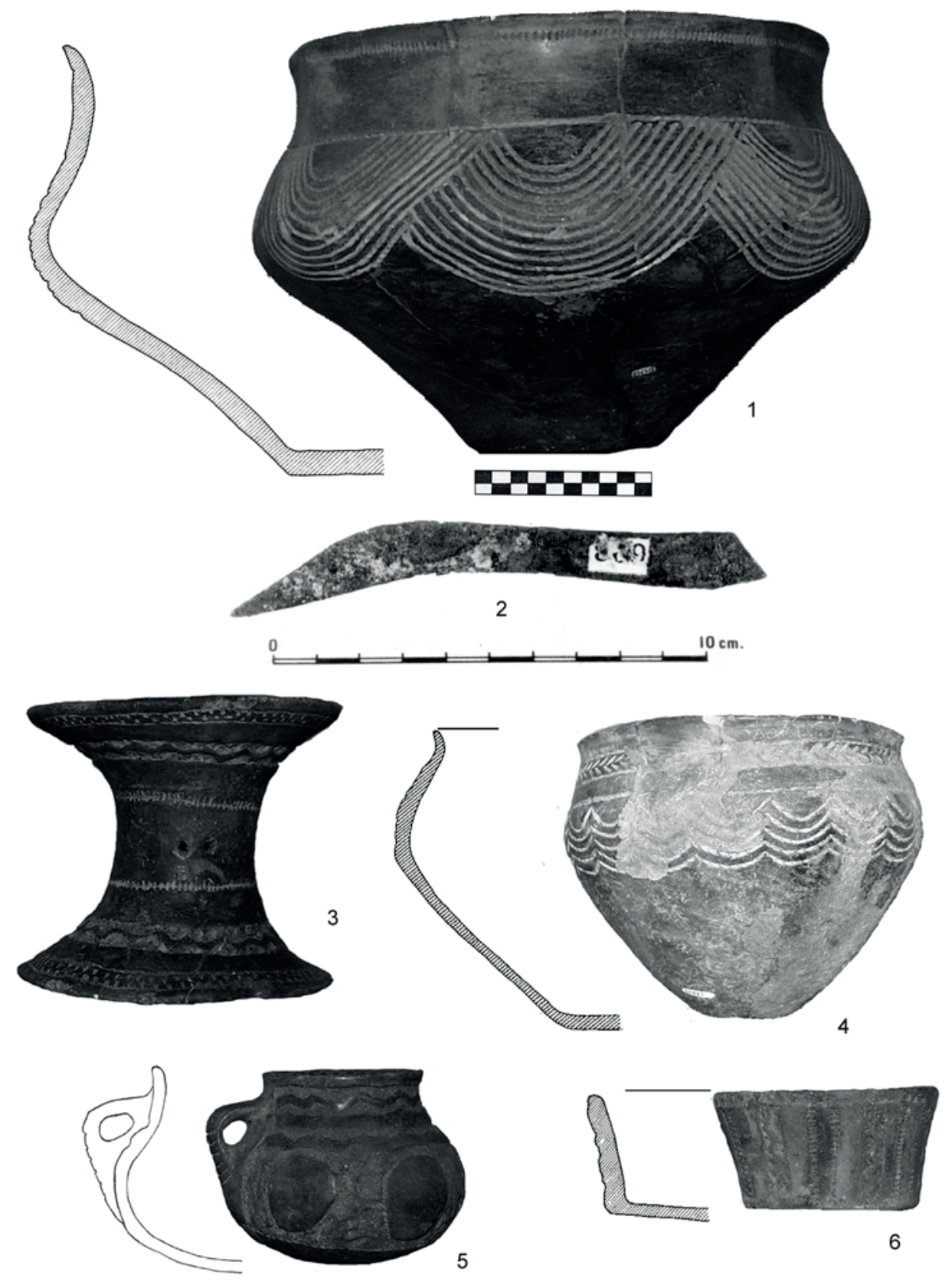

LÁMINA 5: Materiales del Horizonte Cogotas I pertenecientes a la colección Bento (Museo Arqueológico de Barcelona, MAC). 1: Recipiente del Arenero Martínez. 2: Cuchillo de Bronce del Arenero Martínez. 3 a 6 Carrete y recipientes procedentes del Arenero de Valdivia Centro, en su ornamentación se han empleado distintas técnicas. 